\title{
DEFINABLE SETS IN ORDERED STRUCTURES
}

\author{
BY ANAND PILLAY AND CHARLES STEINHORN ${ }^{1}$
}

1. Introduction. We introduce the notion of an O-minimal theory of ordered structures, such a theory being one such that the definable subsets of its models are particularly simple. The theory of real closed fields will be an example. For $T$ an O-minimal theory we prove that over every subset $A$ of a model there is a prime model, which is unique up to $A$-isomorphism. We also prove in our model-theoretic context results on the structure of semialgebraic sets. Our work was directly stimulated by the paper of van den Dries [4].

2. Definitions and examples. $L$ will be a finitary first order language which contains, among other things, a symbol $<$. We shall be concerned with infinite $L$-structures $M$ in which < denotes a linear ordering of $M$. For example if $L$ has symbols $<,+, 0$, then an ordered group is just an $L$-structure $G$ which satisfies the axioms for ordered groups. A definable subset of $M^{n}$ is a subset $X \subset M^{n}$ of the form $\left\{\bar{a} \in M^{n}: M \vDash \varphi(\bar{a}, \bar{m})\right\}$ for some $L$-formula $\varphi(\bar{x}, \bar{y})$ and $m \in M^{r}, r<\omega$. So the definable sets are those which are obtained from the sets defined with parameters from the basic relations and functions on $M$, by closing under finite unions, finite intersections, complementation and projection. An interval of $M$ is something of the form $(a, b),[a, b],(a, b]$ or $[a, b)$, where $a, b \in M$ (or $a=-\infty$, or $b=+\infty$ ). (Such an interval is clearly definable.)

Definition 1. (i) $M$ is $O$-minimal if every definable set $X \subset M$ is a finite union of rational intervals of $M$.

(ii) A complete $L$-theory $T$ is O-minimal if every model of $T$ is O-minimal.

Note that in Definition 1 no condition is placed on the definable subsets of $M^{n}$. An important consequence of the definition is that an O-minimal structure is definably complete; namely every definable subset of $M$ which is bounded above has a supremum in $M$ (and similarly for infimums).

A consequence of the Tarski-Seidenberg theory [3] (i.e. quantifier elimination), is that any real closed field is O-minimal. In fact if $K$ is a real closed field then the definable subsets of $K^{n}, n<\omega$, are precisely the semialgebraic sets over $K$. The following is proved, essentially using the "definable completeness" of O-minimal structures:

THEOREM 2. (i) An ordered group $G$ is O-minimal just if $G$ is abelian and divisible.

(ii) An ordered unitary ring $R$ is $O$-minimal just if $R$ is a real closed field.

Received by the editors January 3, 1984.

1980 Mathematics Subject Classification. Primary $03 C 45$.

${ }^{1}$ Both authors were supported by a grant from NSERC while visiting McGill University, 1982-1983.

(C) 1984 American Mathematical Society $0273-0979 / 84 \$ 1.00+\$ .25$ per page 
Other examples of O-minimal theories (other than the theory of real closed fields and the theory of ordered divisible abelian groups) are the theory of dense linear orderings with no first or last element, and the theory of discrete linear orderings with no first or last element. (This follows from the fact that these theories have quantifier elimination.)

Another important observation is that if $M, N$ are models of an 0-minimal theory $T, M \prec N$ and $a \in N$, then the type of $a$ over $M, \operatorname{tp}(a / M)$ is determined by the cut that $a$ realizes in $M$.

THEOREM 3. If $T$ is $O$-minimal and $\kappa>|T|$, then $T$ has at most one model of cardinality $\kappa$ which is $\kappa$-saturated with respect to the quantifier free language of order.

The theorem of Erdös, Gillman and Henriksen [1] on the uniqueness of a real closed field of cardinality $\aleph_{\alpha}$ and order type $\eta_{\alpha}, \alpha>0$, follows from Theorem 3.

\section{Definable functions.}

LeMMA 4. Let $M$ be $O$-minimal. Let $a, b \in M$ and let $f:(a, b) \rightarrow M$ be $a$ definable function. Then there are $a=a_{0}<a_{1}<\cdots<a_{n}=b$ such that on each $\left(a_{i}, a_{i+1}\right) f$ is either constant or strictly monotone and continuous.

Lemma 4 was proved by van den Dries [4] in the special case where the underlying order on $M$ is that of $\mathbf{R}$. The general case relies again on being able to use the definable completeness of $M$ in place of the completeness of $\mathbf{R}$.

For $a \in M, A \subset M$ we say $a \in \operatorname{cl}(A)$ if there is a function $f(\bar{x})$ definable without parameters such that $f(\bar{b})=a$ for some $\bar{b}$ from $A$. Lemma 3 implies that if $M$ is $O$-minimal, then $b \in \operatorname{cl}(A \cup\{a\}), b \notin \operatorname{cl}(A) \Rightarrow a \in \operatorname{cl}(A \cup\{b\})$. As usual one extracts a notion of independence and dimension: if $A, B \subset M$, $\operatorname{dim}(A / B)=$ the cardinality of a maximal subset $A^{\prime} \subset A$ which is independent over $B$.

4. Existence and uniqueness of prime models. If $A \subset M, M$ is said to be prime over $A$ if for any $N \supset A$, with $(N, a)_{a \in A} \equiv(M, a)_{a \in A}$, there is an elementary embedding $F: M \rightarrow N$ which fixes $A$ pointwise. For example if $k \subset K$ are ordered fields and $K$ is the real closure of $k$ then $K$ is prime over $k$.

Theorem 5. Let $T$ be $O$-minimal, $M=T$ and $A \subset M$. Then there is a model $N$, with $A \subset N \prec M$ such that $N$ is prime over $A$. Moreover $N$ is unique up to $A$-isomorphism.

An important part of the proof of uniqueness is: if $N$ is prime over $A$, $a, b \in N, a, b \in \operatorname{cl}(A),(a, b) \cap \operatorname{cl}(A)=\varnothing$, then $\operatorname{dim}((a, b) / A) \leq \aleph_{0}$.

We should note that if $K$ is a real closed field and $k \subset K$, then $\operatorname{cl}(k)$ is just the field-theoretic algebraic closure of $k$ in $K$, and so $K$ prime over $k$ means $K=\operatorname{cl}(k)$. In this case there do not exist $a, b \in \operatorname{cl}(k)$ with $(a, b) \cap \operatorname{cl}(k)=\varnothing$.

From the point of view of model theory, a strong assumption such as $\mathrm{O}$-minimality is required to guarantee the existence of prime models. For 
example, let $P_{1}, P_{2}$ be unary predicates on $\mathbf{Q}$ such that both $P_{1}, P_{2}$ are dense in $\mathbf{Q}$ and $P_{1} \cup P_{2}=\mathbf{Q}$. Let $M$ be the resulting expansion of $(\mathbf{Q},<)$ and let $A=P_{1}^{M}=$ elements of $M$ in $P_{1}$. Then there is no prime model over $A$. Note $M$ is not $\mathrm{O}$-minimal, as for example the definable set $P_{1}^{M}$ is not of the required form.

Another model-theoretic result is

TheOREM 6. Let $T$ be $O$-minimal and $\aleph_{0}$-categorical. Then $T$ is finitely axiomatisable.

In fact we have described completely the $\aleph_{0}$-categorical 0 -minimal theories. The main point is that $\aleph_{0}$-categoricity precludes the existence of any nontrivial definable function of more than one variable.

5. Decomposition. Here we assume the underlying order to be dense with no first or last element. By definition the definable sets (of elements) in models of an O-minimal theory have a simple structure. Abstractly, we would like to deduce a nice structure for the definable sets of $n$-tuples (i.e. definable sets $\left.X \subset M^{n}, M=T\right)$. In the case of $(\mathbf{R},<,+, \cdot)$ this reduces to studying the structure of the semialgebraic sets, a well-known enterprise $[2,5]$. Our main point is that known results in this special case can be proved in our general model-theoretic context, in particular without the specific use of algebraic, (e.g. differential) structure. The possibility of such proofs was raised by van den Dries [4], whose work we extend.

TheOREM 7. Let $T$ be $O$-minimal and $M$ a model of $T$. Let $\varphi\left(x_{1}, \ldots, x_{n}\right.$, $\left.y_{1}, \ldots, y_{r}\right)$ be an $L$-formula. For each $\bar{m} \in M^{r}$ let $X_{\bar{m}}=\left\{\bar{a} \in M^{n}: M=\right.$ $\varphi(\bar{a}, \bar{m})\}$ (So $\left\{X_{\bar{m}}\right\}_{\bar{m} \in M^{r}}$ is a family of uniformly definable subsets of $M^{n}$.) Then there is a uniform finite bound on the number of connected components of the $X_{\bar{m}}$ as $\bar{m}$ ranges over $M^{r}$.

What is the meaning of connected here? If $M$ is an expansion of $(\mathbf{R},<)$ we can mean the usual topological notion. Otherwise, we will say that a definable subset $X$ of $M^{n}$ is connected if $X$ is not the disjoint union of two nonempty definable, open subsets of $X$. The content of the theorem is that any definable subset of $M^{n}$ is the union of a finite number of nice definable subsets of $M^{n}$-which we call $n$-cells-each of these being connected. Moreover an $n$ cell is defined in a particular syntactic manner using definable functions. For example, a typical 2-cell has the form $\{(x, y): a<x<b, f(x)<y<g(x)\}$, where $a<b \in M$ and $f, g$ are continuous definable functions on $(a, b)$ such that $f(x)<g(x) \forall x \in(a, b)$. To obtain the uniform bound as we vary the parameters, the (model-theoretic) compactness theorem is applied.

\section{REFERENCES}

1. P. Erdös, L. Gillman and M. Henriksen, An isomorphism theorem for real closed fields, Ann. of Math. (2) 61 (1955), 542-554.

2. J. Mather, Stratifications and mappings, Dynamical Systems (M. Peixoto, ed.), Academic Press, 1973, pp. 195-232.

3. A. Tarski and J. C. C. McKinsey, A decision method for elementary algebra and geometry, Rand Corporation, Santa Monica, 1948. 
4. L. van den Dries, Remarks on Tarski's problem concerning $(\mathbf{R},+, \cdot, \exp )$, Proc. Peano Conf. (Florence, 1982) (to appear).

5. H. Whitney, Elementary structure of real algebraic varieties, Ann. of Math. (2) 66 (1957), 545-556.

Department of Mathematics, University of Notre Dame, Notre Dame, INDIANA 46556

Department of Mathematics, Vassar College, Poughkeepsie, New York 12601 